\title{
Enhanced Power Saving Scheme for IEEE 802.11 DCF Based Wireless Networks ${ }^{\star}$
}

\author{
Jong-Mu Choi, Young-Bae Ko, and Jai-Hoon Kim \\ Graduate School of Information and Communication \\ Ajou University, Republic of Korea \\ jmc@dmc.ajou.ac.kr \\ \{youngko, jaikim\}@ajou.ac.kr
}

\begin{abstract}
Providing energy efficiency in MAC (Medium Access Control) layer, while achieving desirable throughput, is an important research issue in the area of wireless networking. A wireless LAN such as IEEE 802.11 using the Distributed Coordination Function (DCF) also provides a mechanism for power conservation which allows each node to "sleep" for some amount of periods, but also requires the nodes to wake up periodically and stay "awake" for a certain duration called the ATIMWindow. In this paper, we propose a new energy-efficient MAC protocol that allows the nodes to go to sleep early, without the need to be "on" for the whole ATIM interval, in the case they are acknowledged that no data is buffered within an ad hoc network and therefore no data transmission will be taken place.
\end{abstract}

\section{Introduction}

A key characteristic of wireless hosts is their operation with a limited energy resource, like batteries in general. Because battery capacity is quite restricted, the issue of reducing power consumption in all aspects of wireless host design has been an important challenge to lengthen battery lifetime. Along with many approaches addressing energy conserving issue at various layers of the network protocols stack, this paper studies on the design of an energy-efficient medium access control (MAC) protocol for wireless networks, especially for IEEE 802.11based wireless LANs.

Past work on energy-efficient MAC protocols can generally be categorized into two: power-control approaches and power-saving approaches. The first approach, so called power-control mechanism, is to adjust the transmission power level for the purpose of energy saving[1]. The main objective of these power control schemes is to find an optimal power level for successfully transmitting data with the minimum transmission power consumption. Another approach for energy-efficient MAC is often named as power-saving schemes because they try

\footnotetext{
* This work is supported by grant No. R05-2003-000-10607-0 from Korea Science \& Engineering Foundation, and grant for upcoming research program of the Korea Research Foundation(KRF-2002-003-D00262).
} 


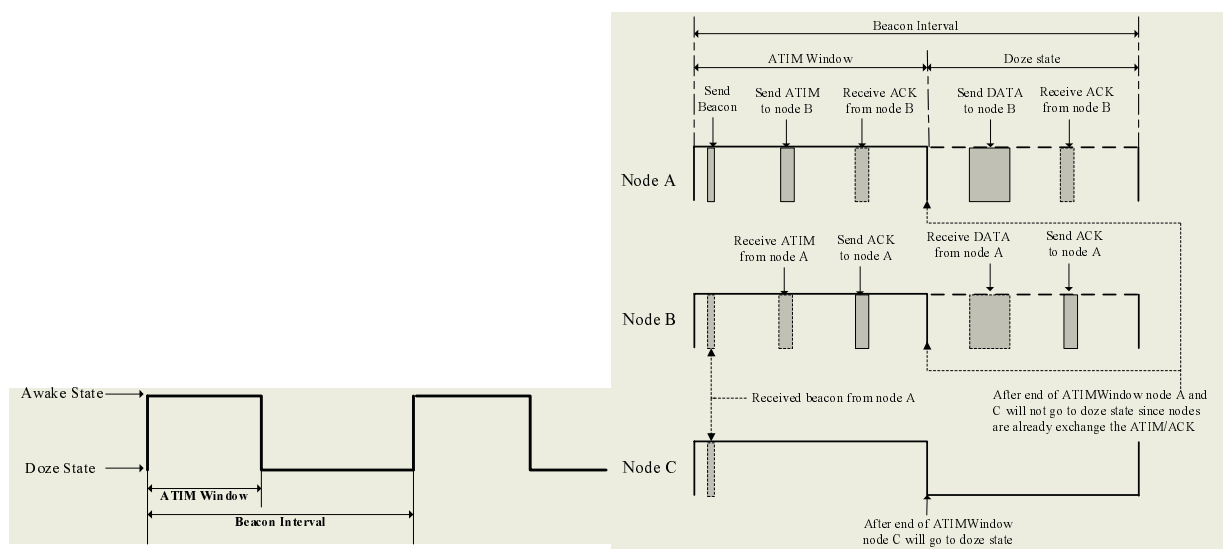

(a) Two operation states

(b) Data exchange

Fig. 1. Operation in IEEE 802.11 DCF (PSM) mode

to achieve energy efficiency by powering off each node's wireless network interface in a certain amount of time 23] whenever needed. Thus, in the power saving mechanism, the wireless hosts are allowed to enter a "sleep" mode by putting their wireless interfaces in a doze state. The IEEE 802.11, standard for WLANs (Wireless Local Area Networks) 4], also provides a similar power saving mechanism using the doze-state and results in some energy conservation by allowing a node to be awake only for certain amount of time - this time is so called ATIMWindow and its value is known to affect the network throughput[5]. Clearly, the larger value of ATIMWindow a node has, the less energy saving it can achieve. Since the node has to be powered on for longer period of time with a shorter sleeping time in a doze state.

This paper proposes enhanced power saving scheme for IEEE 802.11 DCF (Distributed Coordination Function) mode. The new scheme, presented in this paper, guarantees the energy efficiency without degradation of throughput by utilizing the traffic information. According to our proposed scheme, the node can vary the duration of awake-state to adapt the traffic on the network.

\section{Background and Motivation}

In this section, we summarize the PSM (Power Saving Mode) in IEEE 802.11 DCF standard and present a motivation for our work by pointing out some limitation of the standard.

\subsection{Power Saving Mechanism in IEEE 802.11 DCF}

The basic approach of the power saving mechanism in IEEE 802.11 DCF is to periodically change power state between awake and doze, as shown in Fig. 1(a). The figure also shows two terms of "Beacon interval" and "ATIMWindow (Ad hoc Traffic Indication Message Window)". The beacon interval means a fixed 
interval for changing state to awake (i.e., all nodes must be awake at the beginning of every beacon interval) and the ATIMWindow means a fixed awake-period during each beacon interval. Note that, each node should be powered on during an ATIMWindow interval for any possible packet communication (beacon or ATIM). To achieve the change between awake and doze state periodically, all nodes need to be synchronized with their neighbors. For the synchronization, the IEEE 802.11 provides TSF (Timing Synchronization Function), which uses a beacon to notify the time stamp. Every node in DCF is responsible for generating a beacon at the beginning of every beacon interval, and each beacon contains the node's local timestamp. When beacon interval begins, all nodes compete for transmission of their beacon using the standard back-off algorithm. As an example lets assume that there are three nodes competing for sending their own beacons (see Fig. 1(b)). Here node A wins the competition so transmits its beacon. Upon receiving the beacon sent by node A, all other receiving nodes (node $\mathrm{B}$ and $\mathrm{C}$ ) should cancel their beacon transmission schedule and adjust their local timer to the timestamp of the received beacon (i.e., node A's time value).

Fig. 1(b) also shows how data packets can be exchanged in IEEE 802.11 DCF power saving mode. In the figure node A is assumed to have data packets to unicast for node B and therefore transmits its ATIM packet to node B in ATIMWindow. Once the ATIM packet is successfully received, node B may acknowledge to node A with its ACK packet. Successful exchange of ATIM-ACK packets between two nodes implies that they can now exchange any buffered data packets and thus both nodes should stay awake until their data communication completes (i.e., until the next beacon interval). However, all other nodes that are not involved in the exchange phase of ATIM-ACK packets (Node $\mathrm{C}$ in our example) are allowed to enter a doze mode to sleep at the end of ATIMWindow so they can save their energy during the remaining beacon interval.

\subsection{Motivation}

As explained earlier the current IEEE 802.11 standard has a fixed value of ATIMWindow (and a beacon interval), and thus every node must stay awake for the same duration no matter how long to be actually involved in real data communication. Remind that, in the previous Fig. 1,(b), node $\mathrm{C}$ had to stay awake for the whole ATIMWindow unnecessarily because it is not allowed to turn its radio off until this fixed awake duration. It is not difficult to imagine that such inflexibility can cause all nodes to awake and just waste their energies in the worst case when no nodes have data packets to send. We believe that one of the reasons causing this problem is not to consider the actual traffic information in the network. This observation leads us to propose a new energy-efficient MAC protocol that allows the nodes to go to sleep early in the case they are acknowledged that no data is buffered within an ad hoc network and therefore no data transmission will be taken place. 


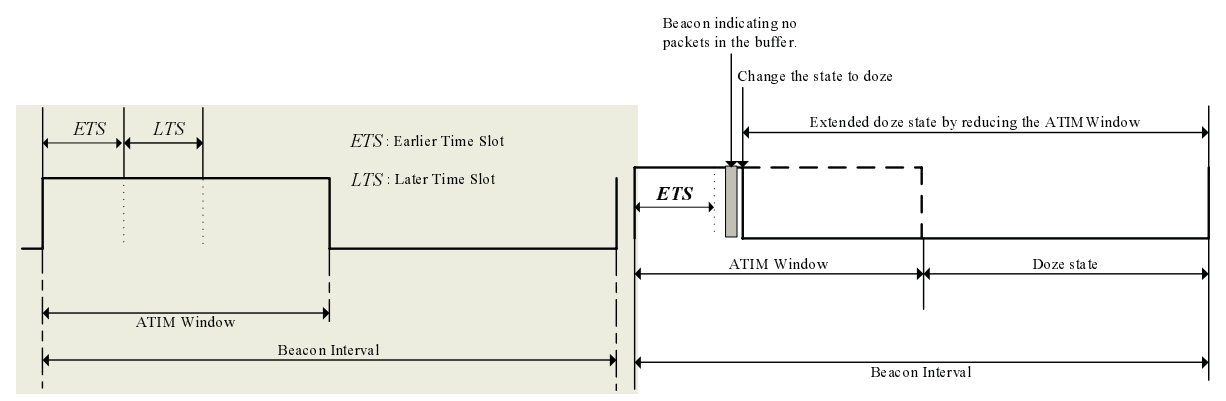

(a) Two time slots for beacon transmission

(b) ATIMWindow size reduction

Fig. 2. Operation of TIPS

\section{Proposed Power Saving Scheme}

In this section, we present a basic idea of our proposed power saving scheme, TIPS (Traffic Indication-based Power Saving). The TIPS protocol is identical to PSM of IEEE 802.11 DCF, with some modification. We will explain these modifications by using the following example in Fig. 2.

In contrast to the original IEEE 802.11 DCF (where a beacon is used for a synchronization purpose only), the basic idea of TIPS is to give one more functionality on a beacon as a future data transmission indicator. To achieve this idea, we made one modification of varying the time point of a beacon transmission based on the beacon transmitter's buffer status. Thus, if the transmitter issuing a beacon has any data packet to send in its buffer, it tries to transmit the beacon in the earlier time slot(ETS in Fig. 2(a)), otherwise, it tries to delay the transmission time in the later time slot (LTS in Fig. 2(a)). If no nodes transmit any beacon in the ETS, all nodes can assume that there are no nodes currently buffering data to send within a present beacon interval. This means that all nodes are allowed to change their state into a doze mode, right after receiving a beacon in the LTS (see Fig. 2(b)). Therefore, in the TIPS, any node buffering data packet must try to send its beacon in the ETS duration.

One of the important issues is how to decide an optimal size of ETS and LTS. There may be several approaches, but in this paper, we propose to use $a C W \max$ value, which is used in the standard random backs off algorithm of IEEE 802.11 as the maximum delay before issuing a beacon. Thus, each node having data packets backoff for a random time chosen from the interval [0,aCWmax] before transmitting its beacon just like the IEEE 802.11 standard. However, this interval becomes $[a C W \max , 2 \times a C W \max ]$ for some nodes having no data packet in their buffer - note that ATIMWindow size is larger than doubled $a C W \max$ value. 


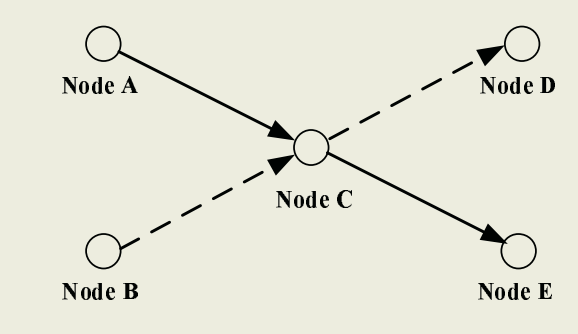

Fig. 3. Topology used in our simulation

\section{Performance Evaluation}

In this section we evaluate the performance of our TIPS, compared to the IEEE 802.11 PSM. For our simulations we have modified the CMU wireless extended version of ns-2.

\subsection{Simulation Model}

The objective of our proposed algorithm is to reduce the energy consumption during the idle time. Thus we use percentage of sleep mode as performance metric.

Fig. 3. shows a simple topology used for our preliminary simulation study. It is a two-hop network with two sources (node A and B) and two destinations (node $\mathrm{D}$ and $\mathrm{E}$ ). Packets from node $\mathrm{A}$ and $\mathrm{B}$ are delivered through node $\mathrm{C}$ to node $\mathrm{D}$ and E, respectively. Each nodes are separated by 200 meters and transmission range of each node is 250 meters. We assume that the maximum bit rate of channel is $2 \mathrm{Mbps}$ and packet size is 512 Bytes. The value of beacon interval is 0.1 seconds. For measuring energy consumption, we use $1.4 \mathrm{~W}, 0.95 \mathrm{~W}, 0.805 \mathrm{~W}$, and $0.06 \mathrm{~W}$ as value of power consumed by the MAC layer in transmit, receive, idle, and doze state respectively. In our simulation, after delivering 10 messages, we measure energy consumption and percentage of sleep time of two schemes (TIPS and PSM).

We vary the traffic load by having different inter-arrival period of the message issued from upper layer (from 0.1 to 1 seconds). We also vary the value of ATIMWindow to observe the effect of its size (from 40 to $80 \mathrm{~ms}$ ).

\subsection{Preliminary Simulation Result}

Fig. 4(a) shows the percentage of sleep time when the ATIM window is $40 \mathrm{~ms}$. When there is little traffic, each node has more chance to make a longer sleep. However, by adjusting sleep time according to traffic information, TIPS always has more sleep time than PSM. This is because, when the traffic is sparse, the

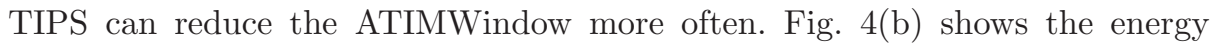
consumption ratio between TIPS and PSM when varying the ATIMWindows and message inter-arrival. This value can be measured by dividing the energy 


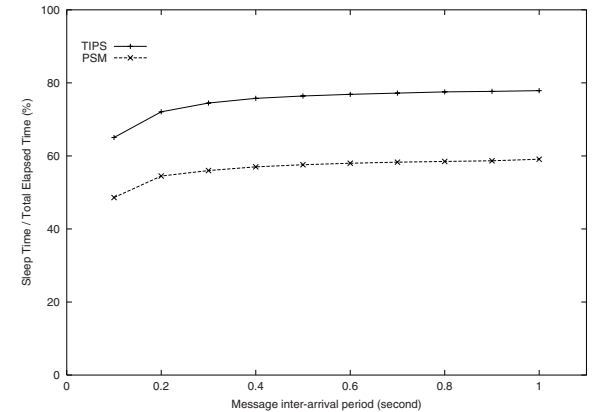

(a) Percentage of sleep time

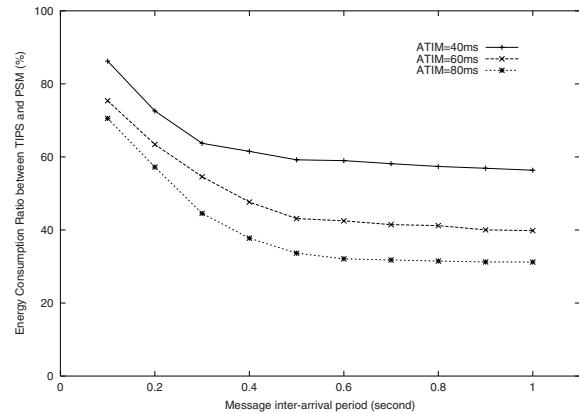

(b) Energy consumption ratio (TIPS/PSM)

Fig. 4. Simulation results

consumption of TIPS to that of PSM. According to the figure, when the network traffic becomes sparse and ATIMWindow is increased, TIPS is more efficient on the aspect of energy consumption. For instance, when the message-interarrival period is equal to 1 the energy improvement of TIPS against PSM is at least $40 \%$ (when ATIMWindow $=40 \mathrm{~ms}$ ).

\section{Conclusions}

In this paper, we propose an enhanced power saving scheme for IEEE 802.11 DCF by utilizing traffic information. To verify the excellence of proposed scheme, we have done some preliminary simulation study, and made very positive results of producing over $70 \%$ energy save in our TIPS compared to the IEEE 802.11 DCF method. Future works include further simulation studies with more realistic environments such as multihop scenarios.

\section{References}

1. E.-S. Jung and N. H. Vaidya, "A Power Control MAC Protocol for Ad Hoc Networks," ACM Mobicom 2002, Oct. 2002.

2. E.-S. Jung and N. H. Vaidya, "An Energy Efficient MAC Protocol for Wireless LANs," IEEE INFOCOM 2002, Jun. 2002.

3. Y.-C. Tseng, C.-S. Hsu, and T.-Y. Heieh, "Power-saving Protocols for IEEE 802.11based Multihop Ad Hoc Networks," IEEE INFOCOM 2002, Jun. 2002.

4. IEEE Computer Society LAN MAN Standard Committee, "Wireless LAN medium access control and physical specification," IEEE 802.11 Standard, Aug. 1998.

5. H. Woesner, J.-P. Ebert, M. Schlager, and A. Wolisz, "Power-Saving Mechanisms in Emerging Standards for Wireless LANs: The MAC Level Perspective," IEEE Personal Communication, Jun. 1998. 